\title{
SUR UNE SÉRIE DE POLYNOMES DONT CHAQUE SOMME PARTIELLE REPRÉSENTE LA MEILLEURE APPROXIMA- TION D'UN DEGRÉ DONNÉ SUIVANT LA MÉTHODE DES MOINDRES CARRES*
}

Par Chamles Jordan.

[Read November 11th, 1920.-Received in revised form February, 18th 1921.]

1. En statistique mathématique on rencontre souvent le problème suivant: étant données certaines valeurs $x_{0}, x_{1}, \ldots, x_{n-1}$ par ordre de grandeur de la variable $x$ auxquelles correspondent respectivement les fréquences $y_{0}, y_{1}, \ldots, y_{n-1}$, le nombre $n$ étant généralement grand, il s'agit de reproduire ces résultats aussi bien que possible à l'aide d'un polynome $f_{m}(x)$ de degré $m$ plus petit que $n$. Les écarts ou erreurs étant $\delta_{i}=y_{i}-f_{l l}\left(x_{i}\right)$, il faut déterminer les coefficients $c_{\nu}$ du polynome $f_{n}(x)=\Sigma c_{\nu} x^{\nu}$ conformément à la théorie des moindres carrés, en rendant la somme des carrés des erreurs $\delta_{i}$ minimum.

Les calculs ne présentent pas de difficultés, mais ils sont longs et pénibles; en effet, les valeurs de $n+1$ déterminants du $m$-ième ordre doivent être calculées.

Les constantes $c_{\nu}$ évaluees, on peut, pour se rendre compte de la précision obtenue, déterminer d'après la théorie des moindres carrés, la somme des carrés des écarts $\delta_{i}$ par la formule suivante:

$$
\Sigma \delta_{i}^{2}=\Sigma y_{i}^{2}-c_{0} . \Sigma y_{i}-c_{1} . \Sigma y_{i} x_{i}-c_{2} . \Sigma y_{i} x_{i}^{2}-\ldots-c_{m} . \Sigma y_{i} x_{i}^{m} .
$$

Si l'on trouve que l'approximation obtenue n'est pas suffisante, pour en avoir une plus grande, on est obligé de refaire le calcul, et de déterminer les coefficients d'un polynome de degré $m_{1}>m$; le grand inconvénient de la méthode est que dans ce cas, tout est à recommencer, car les $m+1$ constantes obtenues précédemment ne conservent pas leurs valeurs.

* L'origine de ce travail est dans un cours de statistique mathématique et de probabilités, que j'ai fait en 1919 ì l'Université de Budapest. 
Si, au lieu de développer le polynome $f_{n 2}(x)$ suivant les puissances de $x$, on fait ce développement suivant les factorielles de $x, x(x-h)$, etc. c.-à-d. si l'on pose:

$$
f_{i m}(x)=\Sigma c_{\nu} x(x-h)(x-2 h) \ldots(x-\nu h+h),
$$

et qu'on détermine les coefficients $c_{\nu}$ d'après le principe des moindres carrés, on rencontre les mêmes difficultés.

Par contre, si l'on fait l'approximation à l'aide d'une série de Fourier, cette difficulté ne se présente pas; en effet dans une telle série les $2 m+1$ constantes étant calculées, si l'on veut obtenir celles d'une série à $2 m_{1}+1$ termes $\left(m_{1}>m\right)$. il suffit de déterminer les constantes supplémentaires, car les premiers $2 m+1$ coefficients restent les mèmes.

Tchehichef a considéré la première fois une série de polynomes tels que le dévelop jement d'une fonction suivant ces polynomes possède la proprièté préciense des séries de Fourier."

Etant donnees les valeurs $y_{0}, y_{1}, \ldots, y_{n-1}$ qu'une fonction $y$ prend pour les valeurs $x_{0}, x_{1}, \ldots, x_{n-1}$ de la variable $x$, il s'agit de determiner une suite de polynomes $\phi_{1}(x), \phi_{9}(x), \ldots, \phi_{m}(x)$, le polynome $\phi_{\nu}(x)$ étant de degré $\nu$, tels que si l'on représente $y$ par la somme de degré $m(m<n)$

$$
f_{m}(. x)=c_{0}+c_{1} \phi_{1}(x)+c_{2} \phi_{3}(x)+\ldots+c_{m} \phi_{m}(x),
$$

la quantitét

$$
\sum_{i=0}^{n}\left[y_{i}-f_{m}\left(x_{i}\right)\right]^{2},
$$

soit minimum pour toutes les valeurs de $m$.

Tchebichef a montré que les polynomes $\phi_{\nu}(x)$ sont proportionnels aux dénominateurs $\psi_{r}(. \cdot r)$ des rédnites de la fraction continue suivante:

$$
\sum_{i=0}^{n} \frac{1}{x-x_{i}}=\frac{a_{1}}{x-b_{1}+\frac{a_{2}}{x-b_{2}+\frac{a_{3}}{x-b_{3}+\ldots}}}
$$

* " Sur une formule d'Analyse," Bull. Phlys. Math. de l'Acadimie Impériale des Sciences de St. Pstersbourg, t. 13 (1854), p. 210 ; "Sur les fractions continues," Journal de mathématiques pures et appliquies, 2 sćrie, t. 3 (1855), p. 289 ; " Sur l'interpolation par la méthode des mnindres carrés," Ménoires de l'Acad. Imp. des Sciences d' St. Pétersbourg, 7 série, t. 1 (1S59), p. 1.

† Dans ce travail, conformément aux principes du calcul des diftérences finies, la variable $x$ ne prend pas la vialeur de la limite supérieure de la somme définie, c.-à-d.

$$
\sum_{x=1}^{n+1} f(x)=f(1)+f(2)+\ldots+f(n)
$$


Le facteur de proportionnalité étant quelconque on peut choisir :

$$
\phi_{\nu}(x)=(-1)^{\nu} \psi_{\nu}(x)\left|\begin{array}{ccccc}
n & \Sigma x_{i} & \Sigma x_{i}^{2} & \ldots & \Sigma x_{i}^{\nu-1} \\
\Sigma x_{i} & \Sigma x_{i}^{2} & \Sigma x_{i}^{3} & \ldots & \Sigma x_{i}^{\prime} \\
\ldots & \ldots & \ldots & \ldots \\
\Sigma x_{i}^{\nu-1} & \ldots & \ldots & \Sigma x_{i}^{2 \nu-2}
\end{array}\right| .
$$

Alors en déterminant les réduites on troure

$$
\phi_{\nu}(x)=\left|\begin{array}{cccccc}
1 & x & x^{2} & x^{3} & \ldots & x^{\prime \prime} \\
n & \Sigma x_{i} & \Sigma x_{i}^{2} & \Sigma x_{i}^{3} & \ldots & \Sigma x_{i}^{\prime \prime} \\
\Sigma x_{i} & \Sigma x_{i}^{2} & \Sigma x_{i}^{3} & \Sigma x_{i}^{4} & \ldots & \Sigma x_{i}^{\nu+1} \\
\ldots & \ldots & \ldots & \ldots & \ldots \\
\Sigma x_{i}^{\nu-1} & \ldots & \ldots & \ldots & \Sigma x_{i}^{2 \nu-1}
\end{array}\right| .
$$

Tchebichef a obtenu la formule suivante donnant les coefficients $c_{\nu}$,

$$
c_{\nu}=\frac{\Sigma y_{i} \phi_{\nu}\left(x_{i}\right)}{\Sigma \phi_{\nu}^{2}\left(x_{i}\right)} .
$$

Dans le mémoire de 1859, mentionné ci-dessus, il a traité en outre le cas particulier dans lequel les valeurs $x_{0}, x_{1}, \ldots, x_{n-1}$ sont équidistantes, dans ce cas entre les fonctions $\phi_{\nu}(x)$ définies dans son mémoire et les polynomes $\psi_{\nu}(x)$ précédents il y a la relation :

$$
\phi_{\nu}(x)=\frac{(2 \nu) !}{\nu !} \psi_{\nu}(x) .
$$

Tchebichef a donné de plus une formule de récurrence pour déterminer les polynomes $\phi_{v}(x)$.

Poincaré dans son Calcul des Probabilités, * a repris la question et il est arrivé, par le développement de $\Sigma 1 /\left(x-x_{i}\right)$ en fraction continue, à des polynomes $D_{\nu}(x)$ proportionnels aux polynomes $\phi_{\nu}(x)$ de Tchebichef.

A. Quiquet, dans les Proceedings of the Fifth International Congress of Mathematicians, Cambridge, + a indiqué une méthode d'application de ces polynomes aux fonctions de survie.

L'emploi de ces polynomes est certainement avantageux, malgré qu'il nécessite la détermination préalable des valeurs de $\phi_{\nu}\left(x_{i}\right)$ et de $\Sigma \phi_{\nu}^{2}\left(x_{i}\right)$ qui 
est généralement laborieuse. Iseur avantage ressort surtout dans le cas où l'on a plusieurs approximations à faire, dans lesquelles les grandeurs $x_{0}, x_{1}, \ldots, x_{n-1}$ sont les mèmes, en effet les valeurs de $\phi_{\nu}\left(x_{i}\right)$, etc., peuvent être calculées alors une fois pour toute. C'est ce qui a lieu si les valeurs de $x_{0}, x_{1}, \ldots$, etc., sont équidistantes, ce qui arrive tr'ès souvent en statistique mathématique.

Dans ce travail nous allons déduire directement la forme générale des polynomes possédant les propriétés mentionnées des séries de Fourier, et étudier leurs propriétés; puis en supposant les valeurs de $x_{i}$ équidistantes, nous allons donner des formules simples et des tables permettant de déterminer les valeurs de ces polynomes; enfin, on va montrer sur im exemple l'emploi de ces formules et de ces tables.

2. Déduction des polynomes. -Etant donnés $n$ points de coordonnées $x_{0}, y_{0} ; x_{1}, y_{1} ; \ldots ; x_{n-1}, y_{n-1}$, soit $y=f_{n-1}(x)$ l'équation d'une courbe de degré $n-1$ passant par ces points. Développons $f_{n-1}(x)$ en une série de polynomes :

$$
y=f_{n-1}(x)=\sum_{\nu=0}^{n} A_{\nu} G_{r,}
$$

oì $A_{\nu}$ est un coefficient constant, $G_{\nu}$ un certain polynome de degré $\nu$.

Considérons une somme partielle de l'expression précédente

$$
y=f_{m}(x)=\sum_{\nu=0}^{m+1} A_{\nu} G_{\nu}
$$

Disposons des coefficients $A_{\nu}$ de manière que lia courbe $y=f_{m}(x)$ de degré $m$ passe aussi près que possible des $n$ points donnés, suivant le principe des moindres carrés c.-à-d. que la somme (2) des carrés des écarts soit minimum :

$$
\sum_{i=0}^{n !}\left[y_{i}-f_{m}\left(x_{i}\right)\right]^{2} .
$$

A cet effet, il faut égaler à zéro les $m+1$ dérivées par rapport i $A_{0}, A_{1}, \ldots, A_{m}$ de cette somme.

Cela nous donne les $m+1$ équations :

pour

$$
\begin{gathered}
\sum_{i=0}^{n}\left[y_{i}-\left(A_{0}+A_{1} G_{1}+A_{2} G_{2}+\ldots+A_{m} G_{m}\right)\right] G_{\nu}=0, \\
\nu=0,1,2,3, \ldots, m .
\end{gathered}
$$

Si les polynomes $G_{\nu}$ sont des polynomes quelconques, les valeurs de $A_{\nu}$ obtenues à l'aide de ces équations dépenderont en général du degré $m$ 
de la courbe. Notre but est de déterminer les polynomes $G_{\nu}$ de manière que les constantes $A_{\nu}$ soient indépendantes de $m$.

La résolution des équations précédentes pour $m=0$ donne :

$$
A_{0}=\Sigma y_{i} / n G_{0}
$$

en remplaçant $A_{0}$ par cette valeur dans les deux équations obtenues de (3) en posant $m=1$, on a $\Sigma G_{0} G_{1}=0$ (équation de condition) et

$$
A_{1}=\Sigma y_{i} G_{1} / \Sigma G_{1}^{2} \text {. }
$$

Si nous posons dans (3) $m=2$ et si nous y remplaçons $A_{0}$ et $A_{1}$ par les valeurs précédentes, nous trouvons :

$$
\Sigma G_{0} G_{2}=0, \quad \Sigma G_{1} G_{2}=0, \quad A_{2}=\Sigma y_{i} G_{2} / \Sigma G_{2}^{2} .
$$

En procédant de la même manière, on arrive aux $\left(\begin{array}{l}n \\ 2\end{array}\right)$ équations de conditions

$$
\sum_{i=0}^{n} G_{\nu}\left(x_{i}\right) G_{\mu}\left(x_{i}\right)=0 \text { pour } \quad \nu \neq \mu .
$$

Les polynomes $G_{0}, G_{1}, \ldots, G_{n-1}$ contiennent en tout $\left(\begin{array}{c}n+1 \\ 2\end{array}\right)$ constantes arbitraires, on peut done satisfaire à ces équations et, en plus, on peut choisir arbitiairement dans chaque polynome un des coefficients.

Outre les equations de condition, on trouve encore:

$$
A_{\nu} \sum_{i=0}^{n}\left[G_{\nu}\left(x_{i}\right)\right]^{2}=\sum_{i=0}^{n} y_{i} G_{\nu}\left(x_{i}\right) .
$$

Par suite, le développement en série de polynomes $G$ possède non seulement l'avantage, vis à vis d'un développement suivant les puissances de $x$, qu'en poussant l'approximation plus loin, les coefficients déjà obtenus conservent leurs valeurs, mais encore l'extrême simplicité de la détermination de ces coefficients $A_{\nu}$.

On peut donner une autre expression aux équations de condition (4); en supposant $\mu>\nu$ on peut mettre à la place de $G_{\nu}$ un polynome quelconque $F_{\nu}\left(x_{i}\right)$ de degré $\nu$. En effet ce dernier peut être considéré comme la somme de plusieurs polynomes $G_{s}$ tels que $s \leqslant \nu$; il en résulte que les conditions (4) sont équivalentes à

$$
\sum_{i=0}^{n} F_{\nu}\left(x_{i}\right) G_{\mu}\left(x_{i}\right)=0 \text { si } \quad \mu>\nu .
$$

Les polynomes mentionnés de Tchebichef et de Poincaré satisfont à cette équation. 
3. Nous rous proposons de résoudre les équations $\left(4^{\prime}\right)$ en supposant les valeurs de $x_{i}$ équidistantes:

$$
x_{i}=a+\left(\frac{b-a}{i n}\right) i=a+h i .
$$

C'est dans ce cas, comme nous l'avons remarqué que l'utilisation de ces polynomes est particulièrement avantageuse. Dans ce cas particulier nous désignerons les polynomes $G_{\nu}$ par $Q_{\nu}$. Pour abréger l'écriture dans la résolution des équations (4'), introduisons une notation nouvelle; les sommes indéfinies de $Q_{m}$ seront désignées, comme il suit :

$$
\Sigma Q_{m} h={ }^{1} Q_{m}, \quad \Sigma \Sigma Q_{m} h^{2}=\Sigma\left(\Sigma Q_{m} h\right) h={ }^{2} Q_{m}, \quad \text { etc., }
$$

de manière que la $\mu$-ième somme indéfinie de $Q_{m}$ sera ${ }^{\mu} Q_{m}$; et la $\mu$-ì'me différence de $Q_{m}$ sera

$$
Q_{m}^{(\mu)}=\Delta^{\mu} Q_{m}
$$

Pour déterminer les polynomes $Q_{m}$, nous allons partir de la somme indéfinie qui correspond à $\left(4^{\prime}\right)$,

$$
\Sigma F_{s}(x) Q_{m}(x) h
$$

En utilisant la méthode de la sommation par parties, de manière ì prendre la somme de $Q_{m}$ et la différence de $F_{s}$, ${ }^{*}$ puis en répetant l'opération $s-1$ fois, jusqu'à arriver it $F_{s}^{(*)}=$ constante, notre somme indéfinie deviendra:

$$
\begin{aligned}
\Sigma Q_{m} F_{s} h={ }^{1} Q_{m} F_{s}-{ }^{2} Q_{m}(x+h) F_{s}^{(1)} & +{ }^{3} Q_{m}(x+2 h) F_{s}^{(2)}-\ldots \\
& +(-1)^{s}{ }^{s+1} Q_{m}(x+s h) F_{s}^{(s)} .
\end{aligned}
$$

Les sommes " $Q_{n}$ ci-dessus ne sont pas complètement déterminées; en effet, on peut leur ajouter sans inconvénient un polynome arbitraire de. degré $v-1$ sans changer $Q_{m}$; nous pouvons donc disposer de ces polynomes arbitraires de manière à annuler les expressions ${ }^{2} Q_{m}(x+\nu h-h)$ pour $x=a$ ou $i=0$ c.-ì-d. pour avoir quel que soit $\nu$,

$$
{ }^{\nu} Q_{m}(a+\nu h-h)=0, \quad \nu \leqslant m .
$$

Il y aura ainsi $m$ conditions et $m$ constantes arbitraires disponibles dans ${ }^{m} Q_{m}$.

$$
\text { * } \Sigma Q(x) F^{\prime}(x) h={ }^{1} Q(x) F^{\prime}(x)-\Sigma^{1} Q(x+h) F^{(1)}(x) h .
$$


Comme dans ces conditions la somme indéfinie précédente est nulle ì la limite inférieure de $\left(4^{\prime}\right)$, pour que cette condition soit satisfaite, il faut que (5) soit aussi nulle à la limite supérieure, c.-à-d. pour $x=b$ ou $i=n$; le polynome $F_{s}$ ainsi que ses différences étant arbitraires, la somme (5) ne peut être nulle pour $x=b$ que si chaque terme est séparément nulle. Il faut done avoir pour toutes les valeurs de $\nu$,

$$
\text { "Q } Q_{m}(b+\nu h-h) h=0 .
$$

De (6) on conclit que l'on a ${ }^{1} Q_{m}(a)=0$, ce qui veut dire que $(x-a)$ doit être un facteur de ${ }^{1} Q_{, m}(x)$ c.-à-d.,

$$
{ }^{1} Q_{m}(x)=(x-a) f_{m}(x) .
$$

De cette relation on arrive, en appliquant la méthode de la sommation par parties, à ${ }^{2} Q_{m}(x)$,

$$
{ }^{2} Q_{m}(x)=\frac{1}{2 !}(x-a)(x-a-h) f_{n}(x)-\Sigma \frac{1}{2 !}(x-a+h)(x-a) f_{m}^{(1)}(x) h .
$$

Pour abréger les formules, nous allons adopter la notation suivante pour la factorielle* :

$$
(x+k)(x+k-h)(x+k-2 h)(x+k-3 h) \ldots(x+k-m h+h)=(x+k)_{m} .
$$

Revenons à notre expression de ${ }^{2} Q_{m}$ et répétons la sommation par parties $m-1$ fois pour avoir :

$$
{ }^{2} Q_{m}(x)=\sum_{\nu=0}^{m+1}(-1)^{\nu} \frac{(x-a+\nu h)_{\nu+2}}{(\nu+2) !} f_{m}^{(\nu)}(x) .
$$

On en conclut que $(x-a)$ et $(x-a-h)$ doivent être des facteurs de ${ }^{2} Q_{m}(x)$ de manière que

$$
{ }^{2} Q_{m}(x)=(x-a)(x-a-h) g_{m}(\dot{x})=(x-a)_{2} g_{m}(x) .
$$

Par sommations successives on démontre de la même manière que

$$
{ }^{m} Q_{m}(x)=(x-a)_{m} \omega_{m}(x) .
$$

Cette grandeur satisfait ì la condition (6) pour qu'elle satisfasse aussi

- Cette notation fait bien ressortir l'analogie entre les puissances et les factorielles; p. ex. on a :

$$
\Sigma(x+k)_{m} h=\frac{1}{m+1}(x+k)_{m+1} \text { et } \int(x+k)^{m} d x=\frac{1}{m+1}(x+k)^{m+1}
$$


ì la condition (7) on part de ${ }^{1} Q_{m}(b)=0$ et en procédant de la mème manière on est conduit à la formule

$$
{ }^{m} Q_{m}(x)=C(x-a)_{m}(x-b)_{m},
$$

$C$ étant un facteur constant arbitraire. Le polynome $Q_{m}$ se trouve donc déterminé, c'est la m-ième différence de l'expression ci-dessus, c.-à-d.,

$$
Q_{n}=C . \Delta^{m}(x-a)_{m}(x-b)_{m} .
$$

En prenant successivement les différences de $C(x-a)_{m}(x-b)_{m}$ on:est conduit sans difficulté à la formule suivante où $\nu \leqslant m$,

(8) $\quad \Delta^{\nu} C(x-a)_{m}(x-b)_{m}=\nu ! h^{\nu} C \sum_{s=0}^{\nu+1}\left(\begin{array}{c}m \\ s\end{array}\right)\left(\begin{array}{c}m \\ \nu-s\end{array}\right)(x-a+s h)_{m-\nu+s}(x-b)_{m-s \cdot}$

L几 même formule peut servir pour les différences d'ordre supérieure à $m$ p. ex. pour $\nu=m+\mu$, mais dans ce cas $s$ ne varie que de $\mu$ à $m+1$; en effet, les termes sous le signe $\Sigma$ sont nuls si $s<\mu$ ou si $s>m$.

Si nous posons $\nu=m$ et $C=1 / 2^{m} . m ! . h^{m}$, les polynomes cherchés deviennent :

$$
Q_{m}(x)=\left(\frac{1}{2}\right)^{m} \sum_{s=0}^{m+1}\left(\begin{array}{c}
m \\
s
\end{array}\right)^{2}(x-a+s h)_{s}(x-b)_{m-s} .
$$

La formule (8) donne les différences des polynomes $Q_{m}$; citons comme exemple :

$$
\Delta^{m} Q_{n}=\frac{(2 m) ! h^{m}}{2^{m} m !} .
$$

De la relation (9) on peut déduire les valeurs des polynomes $Q_{n}$ correspondant à des cas particuliers; p. ex. en posant $a=-1$ et $b=1$, il résulte :

$$
\begin{aligned}
& Q_{0}=1, \\
& Q_{1}=x+\frac{1}{2} h, \\
& Q_{2}=\frac{3}{2} x^{2}+\frac{3}{2} h x+\frac{1}{2} h^{2}-\frac{1}{2}, \\
& Q_{3}=\frac{5}{2} x^{8}+\frac{15}{4} h x^{2}+\frac{\left(11 h^{2}-6\right)}{4} x+\frac{3 h\left(h^{2}-1\right)}{4} .
\end{aligned}
$$

4. Pour pouvoir effectuer les calculs indiqués au commencement du no. 2, il faut encore connaître la valeur de

$$
S_{m}=\sum_{x=a}^{b} Q_{m}^{2}(x) h
$$


On peut déterminer cette somme par la méthode des sommations successives par parties, on trouve un résultat analogue à celui obtenu par Tchebichef dans son mémoire Sur une méthode d'interpolation dejà cité.

$$
S_{m}=\frac{n . h^{2 m+1}}{4^{m}(2 m+1)}\left(n^{2}-1\right)\left(n^{2}-2^{2}\right)\left(n^{2}-3^{2}\right) \ldots\left(n^{2}-m^{2}\right) .
$$

5. Si l'on veut calculer les valeurs des polynomes $Q_{n}$ correspondant à des grandeurs données de $n$ et de $x$, on peut bien se servir de la formule (9), mais il est preférable de déduire d'autres formules plus commodes et plus maniables. Pour y arriver nous allons développer $(x-a)_{m}(x-b)_{m}$ en série de factorielles de $(x-b),(x-b)_{2},(x-b)_{3}$, ... etc., en employant la formule d'interpolation de Newton, qui remplace la formule de Taylor, lorsque au lieu de développer suivant des puissances on veut développer suivant des factorielles. On a :

$$
(x-a)_{m}(x-b)_{m}=\sum_{\nu=0}^{2 m+1} \frac{(x-b)_{\nu}}{\nu ! h^{\nu}}\left[\Delta^{\nu}(x-a)_{n l}(x-b)_{m}\right]_{(x=l)} .
$$

D'après notre formule (8), la $\nu$-ième différence de $(x-a)_{m}(x-b)_{m}$ est égale à zéro pour $x=b$ si $\nu<m$; par contre, si $\nu>m$ cette différence est égale pour $x=b$ à :

$$
\nu ! h^{\nu}\left(\begin{array}{c}
m \\
\nu-m
\end{array}\right)(b-a+m h)_{2 m-\cdot \cdot}
$$

Il résulte de là

$$
(x-a)_{m}(x-b)_{m}=\sum_{\nu-m}^{2 m+1}\left(\begin{array}{c}
m \\
\nu-m
\end{array}\right)(b-a+m h)_{2 m-\nu^{\nu}}(x-b)_{\nu} .
$$

De la même manière en développant $Q_{m}(x)$ suivant les factorielles de $(x-b)$, on aura

$$
Q_{m}(x)=\sum_{\nu=0}^{m+1}\left(\frac{1}{2}\right)^{m}\left(\begin{array}{c}
m \\
\nu
\end{array}\right)\left(\begin{array}{c}
m+\nu \\
\nu
\end{array}\right)(b-a+m h)_{m-\nu}(x-b)_{\nu} .
$$

Comme $(x-a)_{m}(x-b)_{m}$ est symétrique par rapport à $a$ et $b$ et par suite $Q_{m}$ aussi, il existe un développement de $Q_{n}$ en factorielles de $(x-a)$ analogue à l'expression (13); on l'obtient de cette dernière en changeant $a$ en $b$ et inversement.

De (13), on peut déduire directement la différence d'ordre $\mu$ de $Q_{m}$,

$$
\Delta^{\mu} Q_{m}(x)=\left(\frac{1}{2}\right)^{m} \frac{(m+\mu) ! h^{\mu}}{m !} \sum_{\nu=\mu}^{m+1}\left(\begin{array}{c}
m \\
\nu
\end{array}\right)\left(\begin{array}{c}
m+\nu \\
\nu-\mu
\end{array}\right)(b-a+m h)_{m-\nu}(x-b)_{\nu-\mu} .
$$

SER. 2. VOL. 20. No. 1396. 
Voici quelques valeurs particulières de $Q_{m}$ tirées de (13):

$Q_{1}=\frac{1}{2}(b-a+h)+(x-b)$,

$Q_{2}=\frac{1}{4}(b-a+2 h)_{2}+\frac{3}{2}(b-a+2 h)(x-b)+\frac{3}{2}(x-b)_{\leqslant}$,

$Q_{3}=\frac{1}{8}(b-a+3 h)_{3}+\frac{3}{2}(b-a+3 h)_{2}(x-b)+\frac{15}{4}(b-a+3 h)(x-b)_{2}+\frac{5}{2}(x-b)_{3}$.

6. Les polynomes $Q_{n}$ montrent une certaine symétrie. En effet, si dans la formule (9) on remplace $x$ par $a+b-h-x$, on obtient:

$$
Q_{m}=\left(\frac{1}{2}\right)^{m} \sum_{s=0}^{m+1}\left(\begin{array}{c}
m \\
s
\end{array}\right)^{2}(b-x+s h-h)_{s}(a-x-h)_{m-s},
$$

et en changeant le signe de chaque facteur, on trouve

$$
Q_{n}=\left(-\frac{1}{2}\right)^{m} \sum_{s=0}^{m+1}\left(\begin{array}{c}
m \\
s
\end{array}\right)^{2}(x-b)_{s}(x-a+m h-s h)_{m-s},
$$

résultat identique à (9), seul le signe est devenu $(-1)^{n}$; on en conclut

$$
Q_{m}(x)=(-1)^{n} Q_{m}(a+b-h-x) ;
$$

et si nous introduisons une nouvelle variable $x_{1}$ telle que :

$$
x=x_{1}+\frac{1}{2}(a+b-h)
$$

nous aurons

$$
Q_{m}\left(x_{1}\right)=(-1)^{m} Q_{m}\left(-x_{1}\right) \text {. }
$$

Par conséquent, si $m$ est paire, le polynome $Q_{m}$ ne contient que des puissances paires de $x_{1}$; et si $m$ est impaire, $Q_{m}\left(x_{1}\right)$ ne contient que des puissances impaires de $x_{1}$. Dans le cas particulier de $a=-1, b=1$, on a

$$
\begin{aligned}
& Q_{1}=x_{1}, \\
& Q_{2}=\frac{3}{2} x_{1}^{2}+\frac{1}{8} / \iota^{2}-\frac{1}{2}, \\
& Q_{3}=\frac{5}{2} x_{1}^{3}+\frac{1}{8}\left(7 \iota^{2}+12\right) x_{1} .
\end{aligned}
$$

7. Nous allons maintenant introduire au lieu de $x$ une nouvelle variable $\xi$, cette dernière prendra les valeurs entières $0,1,2,3, \ldots, n-1$ définies par la relation

$$
x=a+h \dot{\xi} \quad \text { où } \quad h=\frac{b-\dot{a}}{n} .
$$


Nos formules établies précédemment deviennent:

$$
\begin{gathered}
\dot{q}_{m}=\frac{1}{h^{m}} Q_{m}=\left(\frac{1}{2}\right)^{m} \sum_{\nu=0}^{m+1}\left(\begin{array}{c}
m \\
\nu
\end{array}\right)^{2}(\xi+\nu)_{\nu}(\xi-n)_{n-\nu}, \\
q_{m}=\frac{1}{h^{m}} Q_{m}=\left(\frac{1}{2}\right)^{m} \sum_{\nu=0}^{m+1}\left(\begin{array}{c}
m \\
\nu
\end{array}\right)\left(\begin{array}{c}
m+\nu \\
\nu
\end{array}\right)(n+n)_{m-\nu}(\xi-n)_{\nu}, \\
q_{n}=\frac{1}{h^{m}} Q_{m}=\left(\frac{1}{2}\right)^{m} \sum_{\nu=0}^{m+1}\left(\begin{array}{c}
m \\
\nu
\end{array}\right)\left(\begin{array}{c}
m+\nu \\
\nu
\end{array}\right)(m-n)_{n-\nu}(\xi)_{\nu}, * \\
q_{m}(\xi)=(-1)^{m} q_{m}(n-1-\xi) .
\end{gathered}
$$

La relation $\left(13^{\prime \prime}\right)$ donne immédiatement le développement de $q_{m}(\xi)$ suivant les factorielles de $\dot{\xi}$. [Remarquons que dans ces factorielles, la diffééence étant l'unité, $(\xi)_{\nu}$ signifie $\xi(\xi-1)(\xi-2) \ldots(\xi-\nu+1)$.]

$$
\begin{aligned}
& q_{1}=\frac{1}{2}(1-n)+\xi \\
& q_{2}=\frac{1}{4}(2-n)_{2}+\frac{3}{2}(2-n) \xi+\frac{3}{2}(\xi)_{2}, \\
& q_{3}=\frac{1}{5}(3-n)_{3}+\frac{3}{2}(3-n)_{2} \xi+\frac{15}{4}(3-n)(\xi)_{2}+\frac{5}{2}(\xi)_{3} .
\end{aligned}
$$

En remplaçant dans la formule $\left(13^{\prime \prime}\right)$ les factorielles par les puissances de $\xi$, on trouvet

$$
\begin{aligned}
& 1_{2}=\frac{3}{2} \xi^{2}-\frac{3}{2}(n-1) \xi+\frac{1}{4}\left(n^{2}-3 n+2\right), \\
& \nearrow_{3}=\frac{5}{2} \xi^{3}-\frac{15}{4}(n-1) \xi^{2}+\frac{1}{4}\left(6 n^{2}-15 n+11\right) \xi-\frac{1}{8}\left(n^{3}-6 n^{2}+11 n-6\right), \\
& q_{4}=\frac{35}{8} \xi^{4}-\frac{35}{4}(n-1) \xi^{3}+\frac{5}{8}\left(9 n^{2}-21 n+17\right) \xi^{2}-\frac{5}{8}\left(2 n^{3}-9 n^{2}+17 n-10\right) \xi \\
& +\frac{1}{16}\left(n^{4}-10 n^{3}+35 n^{2}-50 n+24\right) \text {, } \\
& q_{5}=\frac{1.33}{8} \xi^{5}-\frac{315}{16}(n-1) \xi^{4}+\frac{35}{8}\left(4 n^{2}-9 n+8\right) \xi^{3}-\frac{105}{16}\left(n^{3}-4 n^{2}+8 n-5\right) \xi^{2} \\
& +\frac{1}{16}\left(15 n^{4}-105 n^{3}+365 n^{2}-525 n+274\right) \xi \\
& -\frac{1}{32}\left(n^{5}-15 n^{4}+85 n^{3}-225 n^{2}+274 n-120\right) \text {. }
\end{aligned}
$$

* On obtient la formule (13") en partant de (13), si, avant d'introduire la variable $\xi$, on y change $a$ en $b$ et inversement.

† Cette substitution est faite par la formule connue :

$$
(x) m=\sum_{\mu=1}^{m+1}(-1)^{m-\mu} C_{m}^{m-\mu} x^{\mu},
$$

où les coefficients $C_{m i}^{n-\mu}$ sont les nombres de Stirling de première espèce (Voir Nielsen, Gammafunktionen, p. 67). 
8. Maintenant nous sommes en état de pouvoir développer un polynome $F(x)$ de degré $n-1$ en séries de polynomes $Q_{\nu}(x)$, ou un polynome $f(\xi)$ en séries de polynomes $q_{v}(\xi)$ :

$$
F(x)=\sum_{\nu=0}^{n} A_{\nu} Q_{\nu}(x), \quad f(\xi)=\sum_{\nu=0}^{n} a_{\nu} q_{\nu}(\xi)
$$

Pour déterminer les coefficients $A_{v}$ il suffit de multiplier la première équation par $Q_{\nu}$ et de faire la somme des quantités obtenues, $x$ variant de $a$ à $b$, ou $x_{i}$ de $x_{0}$ à $x_{n-1}$. D'après la formule (4), tous les termes du second membre disparaissent sauf le terme en $A_{\nu}$ et l'on trouve :

$$
A_{\nu}=\frac{1}{S_{\nu}} \sum_{i=0}^{n} F\left(x_{i}\right) Q_{\nu}\left(x_{i}\right) \quad \text { ò̀ } \quad S_{\nu}=\sum_{i=0}^{n}\left[Q_{\nu}\left(x_{i}\right)\right]^{2} .
$$

De la méme manière, on aura

$$
a_{\nu} \cdot \sum_{\xi=0}^{n}\left[q_{v}(\xi)\right]^{2}=\sum_{\xi=0}^{n} f(\xi) q_{\nu}(\xi) .
$$

Remarquons en passant que $a_{\nu}=h^{\nu} . A_{\nu}$ et que $q_{\nu} h^{\nu}=Q_{\nu}$.

Si la fonction. $F(x)$ est donnée par $n$ points de coordonnées $x_{0}, y_{0}$; $x_{1}, y_{1} ; \ldots ; x_{n-1}, y_{n-1}$ on peut considérer (1') comme une formule d'interpolation que l'on pourrait appeler formule d'interpolation de Tchebichef; cette formule presente de grands avantages sur les autres formules semblables; les calculs sont plus simples et plus rapides, surtout dans le cas considéré dans ce mémoire où les grandeurs $x_{i}$ sont équidistantes. Alors non seulement on peut utiliser les formules simples que nous venons de donner, mais encore comme nous le verrons, on peut construire des tables abrégeant beaucoup les calculs.

Il n'est pas sans intérêt de comparer les diverses formules d'interpolation :

(1) La formule de Lagrange :

$$
y=\sum_{\nu=0}^{n} y_{\nu} L_{\nu}(x)
$$

où $\quad L_{\nu}(x)=\frac{\omega(x)}{\left(x-x_{\nu}\right)\left[\frac{d \omega}{d x}\right]_{x=x_{\nu}}} \quad$ et $\quad \omega(x)=\left(x-x_{0}\right)\left(x-x_{1}\right) \ldots\left(x-x_{n-1}\right)$.

Si nous considérons une somme partielle de $y$, dans laquelle $\nu$ varie de 0 ì $k$, l'équation obtenue représente une courbe de degré $n-1$, passant 
par les premiers $k$ points de coordonnées $x_{0}, y_{0} ; x_{1}, y_{1} ; \ldots ; x_{k-1}, y_{k-1}$ et par les points de coordonnées $x_{k}, 0 ; x_{k+1}, 0 ; \ldots ; x_{n-1}, 0$.

(2) La formule d'Ampère:

$$
y=y_{0}+\sum_{\nu=1}^{n} B_{\nu}\left(x-x_{0}\right)\left(x-x_{1}\right) \ldots\left(x-x_{\nu-1}\right) .
$$

Les coefficients $B_{\nu}$ sont déterminés successivement en remplaçant $x$ et y par les valeurs correspondantes de $x_{0}, y_{0} ; x_{1}, y_{1} ; \ldots ; x_{n-1}, y_{n-1}$. La somme partielle de $y$, où $\nu$ varie de 0 à $k$, représente une courbe de degré $k-1$ passant par les premiers $k$ points.

(3) Formule de Tchebichef :

$$
y=\sum_{\nu=0}^{\prime \prime} A_{\nu} G_{\nu}(x)
$$

où $A_{\nu}$ est donnée par :

$$
A_{\nu} \sum_{i=0}^{n}\left[G_{\nu}\left(x_{i}\right)\right]^{2}=\sum_{i=0}^{n} y_{i} G_{\nu}\left(x_{i}\right),
$$

et oì $G_{\nu}(x)$ est le dénominateur de la $\nu$-ième réduite de $d / d x[\log \omega(x)]$ développé en fraction continue. La somme partielle de $y$, où " varie de 0 à $k$, représente une courbe de degré $k-1$ passant aussi près des $n$ points donnés que possible selon la théorie des moindres carrés.

Nous avons vu que cette dernière formule ne devient réellement pratique que si les valeurs de $x$ sont équidistantes. Dans ce cas, on a

$$
y=\sum_{\nu=0}^{n} a_{\nu} q_{\nu}(\xi),
$$

où $q_{v}(\xi)$ est donnée par la formule (13"), et $a_{\nu}$ par $\left(16^{\prime}\right)$; la valeur de $s_{\nu}$ est conformément ì la relation (11):

$$
s_{\nu}=\sum_{\xi=0}^{n}\left[q_{\nu}(\xi)\right]^{2}=\frac{n}{4^{m}(2 m+1)}\left(n^{2}-1\right)\left(n^{2}-2^{2}\right) \ldots\left(n^{2}-\nu^{2}\right) .
$$

En outre, on peut construire une fois pour toutes des tables donnant les valeurs de $q_{\nu}(\xi)$ et $s_{\nu}$.

9. Les tables les plus importantes pour le travail statistique en vue de l'utilisation de nos formules sont les suivantes:

(A) Des tables à double entrée donnant les valeurs de $q_{v}(n, \xi)$; une 
table pour chaupe valeur de $\nu$ variant de 1 à 6 ou tout au plus jusqu's̀ 10. L'interpolation à l'aide de polynomes de degré supérieur à 6 ne se. fait que très rarement. On fera varier dans ces tables $n$ de $v+1$ ì 20 ou à 50 selon les besoins.

Nous avons vu que $\xi$ doit varier de zéro à $n$, mais on peut réduire les tables de moitié en tenant compte de la symétrie des polynomes $q_{\nu}(\xi)$ selon $\left(15^{\prime}\right)$.

Cette formule, comme celle de

$$
\sum_{\xi=0}^{n} q_{\nu}(\xi)=0,
$$

peut servir comme rérification aux calculs des tables. Pour déterminer les valeura numériques de $q_{\nu}(\xi)$ on se servira de la formule $\left(13^{\prime \prime}\right)$.

(B) Une table à double entrée donnant les grandeurs $s_{\nu}(n)$ pour les valeurs de $\nu$ et de $n$ qui figurent dans les tables précédentes. On utiliser la formule (11').

A titre d'exemple, nous avons joint à ce mémoire six tables. Les Tables I-V donnent les valeurs de $q_{\nu}(n, \xi)$ poui $\nu=1,2,3,4,5$ et pour $n$ jusqu'à 20 ; la Tabie VI donne $s_{\nu}(n)$ pour les mèmes valeurs de $\nu$ et de $n$.

Nous allons montrer sur un exemple la facilité avec laquelle les constantes $a_{\nu}$ se déterminent en se servant de ces tábles.

La somme des carrés des erreurs mesurant la précision obtenue est

$$
\begin{aligned}
\Sigma \delta_{\xi}^{2} & =\Sigma\left(y_{\xi}-a_{0}-a_{1} q_{1}-a_{2} q_{2}-\ldots\right)^{2} \\
& =\Sigma y_{\xi}^{2}-2 a_{0} \Sigma y_{\xi}-2 a_{1} \Sigma y_{\xi} q_{1}-\ldots+a_{0}^{2} n+a_{1}^{2} \Sigma q_{1}^{2}+a_{2}^{2} \Sigma q_{2}^{2}+\ldots .
\end{aligned}
$$

En y substituant $\Sigma y_{\xi} q_{v}$ it $a_{\nu}, \Sigma q_{\nu}^{2}$ on trouve :

$$
\Sigma \delta_{\xi}^{2}=\Sigma y_{\xi}^{2}-a_{0} \Sigma y_{\xi} q_{1}-a_{2} \Sigma y_{\xi} q_{2}-\ldots-a_{m} \Sigma y_{\xi} q_{m},
$$

dans ces sommes, $\xi$ varie de 0 à $n$. De la relation

il résulte :

$$
\Sigma y_{\xi} q_{\nu}=a_{\nu} s_{\nu}
$$

$$
\sum_{\xi=0}^{n} \delta_{\xi}^{2}=\sum_{\xi=0}^{n} y_{\xi}^{2}-\sum_{\nu=0}^{m+1} s_{\nu} a_{\nu}^{2}
$$

Notons que tous les termes du second membre sauf le premier sont négatifs, ce qui n'était pas nécessairement vraie dans le cas de la formule, du no. 1 donnant la somme des carrés des erreurs. C'est un point im- 
portant, en effet si l'approximation obtenue à l'aide d'un polynome de legré $m$ est insuffisante, pour avoir une meilleure approximation à l'aide d'un polynome de degré $m+1$, les constantes $a_{0}, a_{1}, \ldots, a_{n i}$ obtenues précédemment conservent leurs valeurs et il suffit de calculer la constante $\iota_{m+1}$. La somme des carrés des erreurs sera diminuée de $a_{m+1}^{2} . s_{m+1}$.

Par suite, en calculant ces termes au cours des calculs on se rend toujours compte de l'approximation déjà obtenue, cela permet de juger, si la nécessité de continuer s'impose.

Les calculs sont si simples et peuvent être exécutés si rapidement que ınème lorsque un développement suivant des puissances de $x$ est nécessaire, il y a avantage $\dot{a}$ passer par les polynomes $q_{v}$.

Les polynomes $q_{v}$ sont utiles non seulement aux statisticiens, mais encore aux physiciens, quand il s'agit d'interpréter par un polynome les résultats numériques des expériences.

10. Nous allons montrer un exemple d'interpolation appuye sur les polynomes $q_{v}$, en partant des donnéess empreuntées à Bowley, Elements of statistics (3-iìme éd., p. 91).

Table des
$\begin{array}{cccr} & \\ \text { Salaires. } & \text { Nombre d'ouvriers. } & \xi & y^{2} \\ 0.50 & 317 & 0 & 100489 \\ 1.00 & 1472 & 1 & 2166784 \\ 1.50 & 1297 & 2 & 1682209 \\ 2.00 & 970 & 3 & 940900 \\ 2.50 & 506 & 4 & 256036 \\ 3.00 & 198 & 5 & 39204 \\ 3.50 & 254 & 6 & 64516 \\ 4.00 & 96 & 7 & 9216 \\ 4.50 & 4 & 8 & 16 \\ 5.00 & 9 & 9 & 81 \\ & \Sigma y=5123 & & \Sigma y^{2}=5259451\end{array}$

Dans l'exemple ci-dessus $a=50$ cents, $h=50$ cents, $n=10$. On en tire immédiatement

$$
a_{0}=\frac{\Sigma y}{n}=512 \cdot 3
$$

Si l'on s'arrêtait à ce terme, la somme des carrés des erreur's serait :

$$
\Sigma \delta_{0}^{2}=\Sigma y_{i}^{2}-a_{0} \Sigma y_{\iota}=2634938 .
$$


Pour déterminer la constante $a_{1}$ écrivons en utilisant la Table I pour: $q_{1}(10, \xi)$ et la Table VI pour $s_{1}(10)$,

\begin{tabular}{lccc}
$\xi$ & $y(\xi)-y(9-\xi)$ & $q_{1}(\xi)$ & $q_{1}(\xi)[y(\xi)-y(9-\xi)]$ \\
0 & 308 & $-4 \cdot 5$ & -1386 \\
1 & 1468 & $-3 \cdot 5$ & -5138 \\
$y$ & 1201 & $-2 \cdot 5$ & $-3002 \cdot 5$ \\
3 & 716 & $-1 \cdot 5$ & -1074 \\
4 & 308 & -0.5 & -154 \\
\hline & & & $\Sigma y q_{1}=-10754 \cdot 5$
\end{tabular}

Il en résulte :

$$
a_{1}=\frac{\Sigma y q_{1}}{\Sigma q_{1}^{2}}=-130 \cdot 857 \quad \text { et } \quad \Sigma \delta_{1}^{2}=\Sigma \delta_{0}^{2}-a_{1} \Sigma y q_{1}=1233015 .
$$

Pour déterminer $a_{2}$ et $\Sigma \delta_{2}^{2}$, on procède de la mème manière:

$\begin{array}{lcrc}\xi & y(\xi)+y(9-\xi) & q_{2}(\xi) & q_{2}(\xi)[y(\xi)+y(9-\xi)] \\ 0 & 326 & 18 & 5868 \\ 1 & 1476 & 6 & 8856 \\ 2 . & 1393 & -3 & -4179 \\ 3 & 1224 & -9 & -11016 \\ 4 & 704 & -12 & -8448 \\ & & & 5 y q_{2}=-8919\end{array}$

On en tire

$$
a_{2}=-7 \cdot 51, \quad \Sigma \delta_{2}^{2}=1166981
$$

Détermination du coeflicient $a_{3}$ et de la somme $\Sigma \delta_{3}^{*}$,

$\begin{array}{lccc}\xi & y(\xi)-y(9-\xi) & q_{3}(\xi) & q_{3}(\xi)[y(\xi)-y(9-\xi)] \\ 0 & 308 & -63 & -19404 \\ 1 & 1468 & 21 & 30828 \\ 2 & 1201 & 52 \cdot 4 & 63052 \cdot 5 \\ 3 & 716 & 46 \cdot 5 & 33294 \\ 4 & 308 & 18 & 5544 \\ & & & 5 y q_{3}=113314 \cdot 5 \\ & a_{3}=5 \cdot 87, & \Sigma \delta_{3}^{2}=500879 .\end{array}$


Détermination du coefficient $a_{4}$ et de la somme $\Sigma \delta_{4}^{2}$,

0

1

2

3

4

$$
y(\xi)+y(9-\xi)
$$

326

1476

1393

1224

704 $q_{4}(\xi)$

189

$-231$

$-178 \cdot 5$

$31 \cdot 5$

189 $q_{4}(\xi)[y(\xi)+y(9-\xi)]$

61614

$$
-340956
$$

$-248650 \cdot 5$

38556

133056

$$
\Sigma y \overline{q_{4}=-356380} \cdot 5
$$

$$
a_{4}=-1 \cdot 13, \quad \Sigma \delta_{4}^{2}=98527 .
$$

Détermination de $a_{5}$ et de $\Sigma \delta_{;}^{2}$,

$\begin{array}{lccc}\xi & y(\xi)-y(9-\xi) & q_{5}(\xi) & q_{5}(\xi)[y(\xi)-y(j-\xi)] \\ 0 & 308 & -472 \cdot 5 & -145530 \\ 1 & 1468 & 1102 \cdot 5 & 1618470 \\ 2 & 1201 & -78 \cdot 5 & -94578 \cdot 75 \\ 3 & 716 & -866.25 & 620235 \\ 4 & 308 & -472 \cdot 5 & -145530 \\ & & & \Sigma y q_{5}=612596 \cdot 25\end{array}$

par suite

$$
a_{5}=0 \cdot 1268, \quad \sum \delta_{5}^{2}=20850 .
$$

Il en résulte que si nous nous arrêtons ì $a_{5}$, l'erreur mojenne $\epsilon$ sera:

$$
\epsilon=\left[\frac{\Sigma \delta^{2}}{n}\right]^{k}=45 \cdot 7 \text {. }
$$

La fonction $y$ cherchée est la suivante :

(a)

$$
y=512 \cdot 3+130 \cdot 4 q_{1}-7 \cdot 51 q_{2}+5 \cdot 87 q_{3}-1 \cdot 13 q_{4}+0 \cdot 12 \pi q_{i},
$$

ou en substituant aux polynomes $q$ leurs développements suivant les puissances de $\xi$, on trouve :

(b) $\quad y=320^{\circ} 54+2144 \cdot 76 \xi-1271^{\cdot} 25 \xi^{2}+280 \cdot 35 \xi^{3}-27 \cdot 45 \xi^{4}+\dot{\xi}^{5}$.

Comme vérification, déterminons à l'aide de la formule $(a)$ et ler Tables I-V les écarts $\delta$ correspondant aux valeurs de $\dot{\xi}=0,1, \ldots, 9$; on : 
très rapidement :

\begin{tabular}{lr}
$\xi$ & \multicolumn{1}{c}{$\delta$} \\
0 & $3 \cdot 54$ \\
1 & $-24 \cdot 05$ \\
2 & $63 \cdot 71$ \\
3 & $-67 \cdot 15$ \\
4 & $-6 \cdot 29$ \\
5 & $79 \cdot 99$ \\
6 & $-68 \cdot 27$ \\
7 & $16 \cdot 35$ \\
8 & $4 \cdot 59$ \\
9 & $-2 \cdot 44$
\end{tabular}

La somme de ces erreurs derrait ètre égale à zéro, et la somme de leur carré à 20810 ; effectivement nous avous $\Sigma \delta=-0.02$ et $\Sigma \delta^{2}=20565$, ce qui prouve que les calculs ont été exécutés arec une précision suffisante.

\section{Partie.}

Propriétés mathématiques des polynomes $Q_{\nu}(x)$.

11. Examinons la limite des polynomes $Q_{\nu}(x)$ lorsque l'intervalle $h$ tend vers zéro. Comme

$$
\lim _{l=0}(x-a)_{m}=(x-a)^{m} \quad \text { et } \quad \lim \quad \frac{\Delta^{m}}{h^{m}} F=\frac{d^{m}}{d x^{m}} F,
$$

on conclut : $\quad \lim _{h=0} Q_{m}(x)=\frac{1}{2^{m} m !} \frac{d^{m}}{d x^{m}}(x-a)^{m}(x-b)^{m}$.

Posons $a=-1, b=1$, nous trouverons $\lim Q_{m}=P_{m} ;$ où $P_{m}$ est le $m$-ième polynome de Legendre.

En égalant dans nos formules (9) et (13) $h$ ì zéro, nous obtenons des expressions donnant les polynomes de Legendre.

$$
\left\{\begin{array}{l}
P_{m}=\sum_{s=0}^{m+1}\left(\begin{array}{c}
m \\
s
\end{array}\right)^{2}\left(\frac{x+1}{2}\right)^{s}\left(\frac{x-1}{2}\right)^{m-s}, \\
P_{m}=\sum_{s=0}^{m+1}\left(\begin{array}{c}
m \\
s
\end{array}\right)\left(\begin{array}{c}
m+s \\
s
\end{array}\right)\left(\frac{x-1}{2}\right)^{*}, \\
P_{m}=\sum_{s=0}^{n+1}(-1)^{m-s}\left(\begin{array}{c}
m \\
s
\end{array}\right)\left(\begin{array}{c}
m+s \\
s
\end{array}\right)\left(\frac{x+1}{2}\right)^{s} .
\end{array}\right.
$$


Si nous remplaçons $x$ dans les formules (18) par cos 2 elles deviennent identiques aux développements des polynomes de Legendre suivant les puissances de $\tan ^{2} \frac{1}{2} \mathrm{~A}$, de $\sin ^{2} \frac{1}{2} \mathrm{~T}$ et de $\cos ^{2} \frac{1}{2} \Omega$ donnés par Dirichlet.*

Remarqnons que l'on a aussi d'après la formule (11),

$$
\lim _{h=0} \sum\left(Q_{m}\right)^{2} h=\int_{-1}^{1}\left(P_{m}\right)^{2} d x=\frac{2}{2 m+1} .
$$

12. Nous allons déduire maintenant une équation aux différences finies, dont la solution est le polynome $Q_{n}$. Désignons $(x-a)_{m}(x-b)_{m}$ par $u_{m}(x)$ et déterminons la première différence de $u_{m+1}(x)$ :

$$
\Delta u_{m+1}(x)=(m+1) h[2 x-m h+h-a-b] u_{m}(x) .
$$

Écrivons la différence de $m$-ième ordre de ce produit en utilisant la formule suivante analogue à celle de Leibnitz

$$
\Delta^{n}[U(x) V(x)]=\sum_{s=0}^{n+1}\left(\begin{array}{l}
n \\
s
\end{array}\right) \Delta^{s} V(x+n h-s h) \Delta^{n-s} U(x),
$$

d'après cette formule on $a$ :

$$
\Delta^{n+1} u_{m+1}=(m+1) h\left[(2 x+m h+h-a-b) \Delta^{m} u_{m}+2 m h \Delta^{m-1} u_{m}\right] .
$$

On peut encore exprimer cette quantité autrement, en considérant $u_{m+1}$ comme le produit des deux facteurs $u_{m}(x)$ et $u_{1}(x-m h)$. On trouve alors à l'aide de (18a),

$$
\begin{aligned}
\Delta^{m+1} u_{m+1}=u_{1}(x+h) \Delta^{m+1} u_{m}+(m+1) \Delta u_{1}(x) \Delta^{m} u_{m}(x) \\
+\left(\begin{array}{c}
m+1 \\
2
\end{array}\right) \Delta^{2} u_{1}(x-h) \Delta^{m-1} u_{m}(x)
\end{aligned}
$$

En remarquant que $\Delta u_{1}(x)=(2 x \dddot{+} h-a-b) h$ et que $\Delta^{2} u_{1}(x)=2 h^{2}$ on tire de (19) et de (20),

$$
u_{1}(x+h) \Delta^{m+1} u_{m}(x)-m(m+1) h^{2} \Delta^{m} u_{m}(x)-m(n+1) h^{2} \Delta^{m-1} u_{m}(x)=0 .
$$

La première différence de cette expression donne, si l'on remplace $\Delta^{m} u_{m}(x)$ par $1 / C . Q_{m}$, l'équation aux différences cherchée:

(21) $(x-a+2 h)(x-b+2 h) \Delta^{2} Q_{m}+[2 x+3 h-m(m+1)-a-b] h . \Delta Q_{m}$

$$
-m(m+1) h^{2} \cdot Q_{n}=0 \text {. }
$$

\footnotetext{
* Crelle Journal, Bd. 17, pp. 39, 40.
} 
Si nous posons $a=-1, b=1$ et si nous faisons tendre $h$ vers zéro, l'equation (21) est transformée en une équation différentielle du second ordre admettant comme solution le polynome de Legendre de degré $m$.

En introduisant la variable $\xi$ au lieu de $x$ d'après (16) on a :

$$
(\xi+2)(\xi-n+2) \Delta^{2} q_{m}+[2 \xi-n+3-m(m+1)] \Delta q_{m}-m(m+1) q_{m}=0 .
$$

La solution de cette équation est donnée par la méthode de Boole (Treatise on Finite Differences, 1860, p. 176),

$$
\begin{gathered}
q_{m}(\xi)=\sum_{\nu=0}^{m+1} b_{\nu}(\xi+\nu)_{\nu}, \\
b_{\nu}=(-1)^{\nu}\left(\begin{array}{c}
m \\
\nu
\end{array}\right)\left(\begin{array}{c}
m+\nu \\
\nu
\end{array}\right) \frac{b_{0}}{(n+\nu)_{\nu}},
\end{gathered}
$$

où $b_{0}$ est une constante arbitraire. De la première de ces deux relations il resulte que $b_{0}=q_{m}(-1)$. En remplaçant $\xi$ par -1 dans notre formule $\left(9^{\prime}\right)$ nous obtenons:

$$
\left\{\begin{aligned}
b_{0} & =\left(-\frac{1}{2}\right)^{m}(m+n)_{m} . \\
\text { et } \quad q_{m} & =\sum_{\nu=0}^{m+1}(-1)^{m-\nu}\left(\frac{1}{2}\right)^{m}\left(\begin{array}{c}
m \\
\nu
\end{array}\right)\left(\begin{array}{c}
m+\nu \\
\nu
\end{array}\right)(m+n)_{m-\nu}(\xi+\nu)_{\nu} .
\end{aligned}\right.
$$

C'est une formule semblable à celle de $\left(13^{\prime}\right)$; elle est aussi très commode pour le calcul des polynomes $q_{m}(\xi)$. En $\mathrm{g}$ remplaçant $n$ par $2 / h$, lq variable $\xi$ par $(x+1) / h$, et $q_{m}(\xi)$ par $Q_{m}(x) h^{m}$ et en faisant tendre $h$ vers zéro, la formule (23) coïncide à la limite avec la troisième formule (18) donnant les polynomes de Legendre.

13. Pour établir l'equation fonctionnelle qui relie les polynomes $Q$ de divers degrés, il suffit de développer $x . Q_{m}$ en séries de polynomes $Q_{m}$; d'après ce que nous avons vu, ce développement ne contient que les trois termes suivants :

$$
x Q_{m}=A_{n-1} Q_{m-1}+A_{v 2} Q_{m}+A_{m+1} Q_{m+1} .
$$

Les autres termes étant nuls conformément ì l'équation $\left(4^{\prime}\right)$; et l'on a :

$$
\begin{aligned}
& A_{m-1}=\frac{1}{S_{m-1}} \sum_{x=a}^{b} x \cdot Q_{m} Q_{m-1} h, \\
& A_{m}=\frac{1}{S_{m}} \sum_{x=a}^{b} x\left(Q_{m}\right)^{2} h, \\
& A_{m+1}=\frac{1}{S_{m+1}} \sum_{x=a}^{b} x \cdot Q_{m} Q_{m+1} h .
\end{aligned}
$$


La détermination des coefficients $A_{m-1}$ et $A_{m+1}$ ne présente pas de difficultés, en répétunt sur (24) la sommation par parties, on est conduit à la grandeur $\Sigma(x+m h-a)_{m}(x+m h-b)_{m}$ que l'on peut évaluer à l'aide d'une formule analogue à celle donnée par Cauchy pour le dévelop pement de $(x+y)_{n}$ en factorielles de $x$ at $y$. On trouve enfin :

$$
A_{m+1}=\frac{m+1}{2 m+1}, \quad A_{m-1}=\frac{m}{2 m+1} \frac{(b-a)^{2}-m^{2} h^{2}}{4} .
$$

La détermination du troisième coefficient, par la même méthode, conduirait à des difficultés. Elle nécessiterait l'évaluation de

$$
\Sigma(x+h m+h-a)_{m}(x+h m+h-b)_{m}
$$

ce qui est difficile, par contre on arrive directement au résultat en remarquant que l'équation (24) doit avoir lieu pour toutes les valeurs de $x$ donc aussi pour $x=b$, mais de (9) il résulte que

$$
Q_{m}(b)=\left(\frac{1}{2}\right)^{m}(b-a+n \iota)_{m} .
$$

Ln remplaçant dans (24) $A_{m-1}, A_{m+1}, Q_{m}(b), Q_{m-1}(b)$, et $Q_{m+1}(b)$ par:les valeurs correspondantes, on peut déterminer la seule inconnue $A_{m}$,

Finalement on a

$$
A_{m}=\frac{1}{2}(a+b-h) \text {. }
$$

$$
4(m+1) Q_{m+1}-2(2 m+1)(2 x-a-b+h) Q_{n}+m\left[(b-a)^{2}-m^{2} h^{2}\right] Q_{m-1}=0 .
$$

En posant $a=-1$ et $b=1$ nous obtenous l'équation de 'l'chebichef mentionnée au no. 1 ; si en outre nous posons $h=0$, l'équation (25) coïlcide avec l'équation bien connue vérifiée par les polynomes de Legendre,

$$
(m+1) P_{m+1}-(2 m+1) x . P_{m}+m . P_{m-1}=0 .
$$

14. La méthode des fonctions génératrices de Laplace appliquée à (2:5) conduit à une équation différentielle dont la solution est la fonction ¿zénératrice des polynomes $Q_{m}$.

En désignant par $G[\psi(m)]$ la fonction génératrice de $\psi(m)$, on a :

$$
G[\psi(m)]=\sum_{m=0}^{\infty} \psi(m) t^{m}
$$

Posons

$$
G\left(Q_{m}\right)=\phi \quad \text { et } \quad \frac{d \phi}{d t}=\phi^{\prime},
$$


nous aurons :

$$
\begin{aligned}
G\left[(m+2) Q_{m+2}\right] & =\frac{1}{t}\left(\phi^{\prime}-Q_{1}\right), & G\left[Q_{m+1}\right] & =\frac{1}{t}\left(\phi-Q_{0}\right), \\
G\left[(m+1) Q_{m+1}\right] & =\phi^{\prime}, & G\left[m Q_{m}\right] & =t \phi^{\prime}, \\
G\left[m^{2} Q_{m}\right] & =t^{2} \phi^{\prime \prime}+t \phi^{\prime}, & G\left[m^{3} Q_{m}\right] & =t^{3} \phi^{\prime \prime \prime}+3 t^{2} \phi^{\prime \prime}+t .
\end{aligned}
$$

En écrivant dans (25) $m+1$ au lieu de $m$ et $x_{1}$ au lieu de $x-\frac{1}{2}(a+b-h)$, on obtient à l'aide des quantités précédentes la fonction génératrice du premier membre de l'equation (25); en égalant cette fonction génératrice à zéro, nous obtenons une équation différentielle linéaire du troisième ordre dont la solution est la fonction génératrice cherchée:

$$
\begin{aligned}
\phi^{\prime \prime \prime} t^{4} h^{2}+6 \phi^{\prime \prime} t^{3} h^{2}+\phi^{\prime} & {\left[\left(7 h^{2}-b^{2}+2 a b-a^{2}\right) t^{2}+8 x_{1} t-4\right] } \\
& +\phi\left[\left(h^{2}-b^{2}+2 a b-a^{2}\right) t+4 x_{1}\right]=Q_{1}-x_{1} Q_{0} .
\end{aligned}
$$

Remarquons que dans le cas particulier des polynomes $Q_{\nu}$, cette équation se simplifie, car $Q_{0}=1$ et $Q_{1}=x_{1} Q_{0}$, donc le second membre de l'équation (26) est nul.

Si nous posons dans (26) $h=0, a=-1, b=1$, l'équation se transforme en une équation différentielle admettant comme solution la fonction génératrice des polynomes de Legendre,

$$
\phi^{\prime}\left(t^{2}-2 x_{1} t+1\right)+\phi\left(t-x_{1}\right)=0 .
$$

On en tire: $\quad \phi=\left(t^{2}-2 x_{1} t+1\right)^{-3}$.

Dans notre cas, il est possible de donner une forme plus simple ì l'équation (26) en posant

et

$$
\begin{gathered}
\phi=t^{-2} \psi \\
\left.\frac{1}{h^{2} t^{4}}:\left[h^{2}-(b-a)^{2}\right] t^{2}+8 x_{1} t-4\right\}=R .
\end{gathered}
$$

Il vient

$$
\psi^{\prime \prime \prime}+\psi^{\prime} R+\frac{1}{2} \psi \frac{d R}{d t}=0
$$

La résolution de cette équation donnerait la fonction génératrice des polynomes $Q_{m}$.

Notes.

Ce mémoire ayant été communiqué à Mr. L. Fejér, il a démontré les propositions suivantes : 
1. Étant donnés $n$ points dont les abscisses sont par ordre de grandeur $x_{0}, x_{1}, x_{2}, \ldots, x_{n-1}$, parmi tous les polynomes $g_{n}(x)$ de degré $m$, dans lesquels le coefficient du terme en $x^{m}$ est l'unité, le polynome $G_{m}(x)$ qui rencl minimum l'expression

$$
\sum_{i=0}^{n}\left[g_{n}\left(x_{i}\right)\right]^{2} \quad(m<n)
$$

est proportionnel au polynome $\psi_{m}$ de Tchebichef, mentionné au no. 1.

Si les différences $x_{i}-x_{i-1}$ sont constantes, $G_{m}(x)$ est proportionnel it notre polynome $Q_{m}(x)$.

Cette proposition présente une analogie avec la suivante: Parmi les polynomes considerés précédemment, celui qui rend minimum l'intégrale

$$
\int_{-1}^{1}\left[g_{n n}(x)\right]^{2} d x
$$

est proportionnel aux polynome $P_{m}$ de Legendre.*

2. Les racines de l'équation $G_{m}(x)=0$ et par suite aussi des équations $\psi_{m}=0$ et $Q_{n}=0$ sont toutes reelles et comprises dans l'intervalle $\left(x_{0}, x_{n-1}\right)$.

3. L'équation $G_{m}(x)=0$ n'a pas de racines multiples et dans tout intervalle $\left(x_{i}, x_{i+1}\right)$ il y a au plus une racine de cette équation.

Pour prouver ces propositions, nous allons poser avec Mr. Fejér :

$$
g_{m}(x)=c_{0}+c_{1} x+c_{2} x^{2}+c_{3} x^{3}+\ldots+c_{m-1} x^{x^{n-1}}+x^{m} .
$$

Proposition I.-En vue de rendre minimum l'expression (28) égalons à zéro les dérivées de cette dernière par rapport aux coefficients $c_{\nu}$,

$$
\sum_{i=0}^{n} x_{i}^{\nu} g_{m}\left(x_{i}\right)=0 \quad\left(0 \leqslant \nu<m_{j}^{\prime} .\right.
$$

Ces conditions sont identiques ì nos conditions (4) qui déterminent les polynomes $\psi_{m}$ et $Q_{m}$, on peut donc définir ces dernières comme rendant minimum l'expression (28).

Proposition II.-Cette proposition est la conséquence immédiate d'un théorème plus général dû à Mr. Fejér. 
Étänt donnés $n$ points $z_{0}, z_{1}, \ldots, z_{n-1}$ dans le plan de la variable complexe $z$, soit $G_{m}(z)$ le polynome de degré $m(m<n)$, dans lequel le coefficient de $z^{m}$ est égal à l'unité, et qui rende minimum l'expression suivante :

$$
\sum_{i=0}^{n}\left|g_{m}\left(z_{i}\right)\right|^{2}
$$

Théorème: Si $a, b, c, \ldots$, sont les racines de $G_{m}(z)=0$, aucune de ces racines ne peut représenter un point exterieur au plus petit polygone convex contersant les points $z_{0}, z_{1}, \ldots, z_{n-1}$.

Démonstration: Supposons que l'une de ces racines, p. ex. $z=a$ corresponde à un point extérieur au polygone mentionné. S'il est possible de déplacer le point $a$ en $a_{1}$ de manière que toutes les distances $\left|z_{i}-a\right|$ diminuent, $(i=0,1, \ldots, n-1)$, c.-à-d. que

$$
\left|z_{i}-a_{1}\right|<\left|z_{i}-a\right|
$$

pour toutes les valeurs de $i$, nous obtiendrons un polynome

$$
H_{n z}(z)=\left(z-a_{1}\right)(z-b)(z-c) \ldots
$$

tel que

$$
\left|H_{m}\left(z_{i}\right)\right|<\left|G\left(z_{i}\right)\right|
$$

pour toutes les valeurs de $i$ pour lesquelles $G\left(z_{i}\right) \neq 0$; aux autres valeurs $H_{m}\left(z_{i}\right)=G\left(z_{i}\right)$, par conséquent on aurait

$$
\Sigma\left|H_{m}\left(z_{i}\right)\right|^{2}<\Sigma\left|G_{n i}\left(z_{i}\right)\right|^{2}
$$

ce qui serait contraire à la supposition que $G_{m l}(z)$ rend minimum l'expression (30).

Il reste encore à montrer que, le point $a$ étant un point extérieur au polygone mentionné ci-dessus, il est effectivement possible de déplacer ce point de manière à diminuer toutes les distances $\left|z_{i}-a\right|$. En effet, si le point $a$ est un point extérieur, il est toujours possible de mener une droite $D$ de manière que le polygone soit situé d'un còté de la droite, et le point $a$ de l'autre. Menons par le point $a$ une perpendiculaire à $D$, si le point a se déplace sur cette perpendiculaire vers la droite $D$, on peut voir aisément que toutes les distances $\left|z_{i}-a\right|$ diminuent; on en conclut qu'aucune des racines de $G_{m}(x)=0$ ne peut être situee en dehors du polygone considéré; la démonstration du théorème est donc complète.

Dans le cas particulier où les $n$ points donnés sont tous situés sur l'axe réel, le polygone est réduit au segment de droite $\left(x_{0}, x_{n-1}\right)$, par suite d'apiès le thérème que l'on vient de démontrer, les racines de $G_{m}(x)$ sont toutes situées entre ces deux points, donc elles sont toutes réelles. 
Proposition III.-Nous allons démontrer qu'entre deux points consécutifs quelconques $x_{i}$ et $x_{i+1}$ le polynome $Y=G_{m}(x)$ ne peut changer de. sigue plus d'une fois.

D'abord dans la suite $Y_{0}, Y_{1}, \ldots, Y_{n-1}$ correspondant $\dot{a} x_{0}, x_{1}, \ldots, x_{n-1}$ la quantité $Y_{i}$ change de signe $m$ fois.

En effet $G_{m}(x)$ étant de degré $m$, il est évident que la série ci-dessus ne peut présenter plus de $m$ changements de signe. Supposons, qu'il $\boldsymbol{v}$ ait moins, p. ex. $\mu(\mu<m)$, dans ce cas il serait possible de mener une courbe de degré $\mu$ soit $y=f_{\mu}(x)$ telle que pour toutes les valeurs de $i$ les grandeurs $y_{i}=f_{\mu}\left(x_{i}\right)$ et $Y_{i}$ aient le même signe, lorsque $y_{i}$ et $Y_{i}$ sont différentes de zéro.

Supprimons les points $x_{i}$ correspondant aux valeurs nulles de $Y_{\boldsymbol{i}}$; supposons qu'en suite les changements de signes de $Y_{i}$ ont lieu entre les points $x_{k}$ et $x_{\ell}$, entre $x_{i l}$ et $x_{r}$, etc., alors la courbe suivante de degré $\mu$,

$$
y=f_{\mu}(x)=(-1)^{\mu} Y_{k}\left(x-\frac{1}{2} x_{k}-\frac{1}{2} x_{p}\right)\left(x-\frac{1}{2} x_{q}-\frac{1}{2} x_{r}\right) \ldots
$$

change de signe au milieu des mèmes intervalles que $Y$, et l'on voit facilement que le signe de $f_{\mu}\left(x_{i}\right)$ et $Y_{i}$ est le même pour toutes les valeurs de $i$ pour lesquelles $y_{i}$ et $Y_{i}$ sont différentes de zéro. Or il y a de telles valeurs, car d'après notre supposition $Y_{l i}$ est différente de zéro et à cause de (31) $y_{k}$ l'est aussi. On en conclut que

$$
\sum_{i=0}^{n} f_{\mu}\left(x_{i}\right) G_{m}\left(x_{i}\right)>0,
$$

ce qui contredit notre condition (29). Le polynome $G_{m}(x)$ ne rendrait pas l'expression (30) minimum, donc en supposant qu'il y a moins de $m$ changements de signe dans la suite $Y_{0}, Y_{1}, \ldots, Y_{n-1}$ on arrive à une contradiction.

Ainsi nous avons démontré que le polynome $G_{m}(x)$ change de signe $m$ fois entre $x_{0}$ et $x_{n-1}$, comme il est de degré $m$ toutes ces racines sont simples. La première partie du théorème est donc démontrée. Pour montrer qu'entre deux racines consécutives de $G_{m}(x)=0$ se trouve placé au moins un des points $x_{i}$, il suffit de remarquer que dans le cas contraire la suite $Y_{0}, Y_{1}, \ldots, Y_{n-1}$ présenterait nécessairement moins de $m$ changements de signe, ainsi dans l'intervalle $x_{i} \leqslant x \leqslant x_{i+1}$ il y a au plus une racine de $G_{m}(x)=0$.

En résumé : étant donnés $n$ points, dont les abscisses sont par ordre de grandeur: $x_{0}, x_{1}, \ldots, x_{n-1}$, parmi tous les polynomes $g_{m}(x)$ de degré $m$, dans lesquels le coefficient de $x^{n 2}$ est l'unité, soit $G_{m}(x)$ le polynome qui

SER. 2. VOL. 20 . NO. 1397. 
rend minimum l'expression:

$$
\sum_{i=0}^{n}\left[g_{m}\left(x_{i}\right)\right]^{2} \quad(m<n) .
$$

Le polynome $G_{m}(x)$ est proportionnel au polynome $\psi_{m}$ de Tchebichef, de plus toutes les racines de ce polynome sont réelles et comprises entre $x_{6}$ et $x_{n-1}$; en outre, cette équation n'a pas de racine multiple et parmi les $n-1$ intervalles il y a $m$ intervalles $\left(x_{i}, x_{i+1}\right)$ renfermant une racine de $G_{m}(x)=0$.

Dans le cas particulier où $m=n-1$, chacun des intervalles mentionnés contient une racine.

I. TABLE DES Valeurs DE $\eta_{1}(n, \xi)$.

\begin{tabular}{|r|l|l|l|l|l|l|l|l|l|l|l|l|}
\hline$n, \xi$ & 0 & 1 & 2 & 3 & 4 & 5 & 6 & 7 & 8 & 9 & 10 & 11 \\
\hline 2 & -0.5 & 0.5 & & & & & & & & & & \\
3 & -1 & 0 & 1 & & & & & & & & & \\
4 & -1.5 & -0.5 & 0.5 & 1.5 & & & & & & & & \\
5 & -2 & -1 & 0 & 1 & 2 & & & & & & & \\
6 & -2.5 & -1.5 & -0.5 & 0.5 & 1.5 & 2.5 & & & & & & \\
7 & -3 & -2 & -1 & 0 & 1 & 2 & 3 & & & & & \\
8 & -3.5 & -2.5 & -1.5 & -0.5 & 0.5 & 1.5 & 2.5 & 3.5 & & & & \\
9 & -4 & -3 & -2 & -1 & 0 & 1 & 2 & 3 & 4 & & & \\
10 & -4.5 & -3.5 & -2.5 & -1.5 & -0.5 & 0.5 & 1.5 & 2.5 & 3.5 & 4.5 & & \\
11 & -5 & -4 & -3 & -2 & -1 & 0 & 1 & 2 & 3 & 4 & 5 & \\
12 & -5.5 & -4.5 & -3.5 & -2.5 & -1.5 & -0.5 & 0.5 & 1.5 & 2.5 & 3.5 & 4.5 & 5.5 \\
13 & -6 & -5 & -4 & -3 & -2 & -1 & 0 & 1 & 2 & 3 & 4 & 5 \\
14 & -6.5 & -5.5 & -4.5 & -3.5 & -2.5 & -1.5 & -0.5 & 0.5 & 1.5 & 2.5 & 3.5 & 4.5 \\
15 & -7 & -6 & -5 & -4 & -3 & -2 & -1 & 0 & 1 & 2 & 3 & 4 \\
16 & -7.5 & -6.5 & -5.5 & -4.5 & -3.5 & -2.5 & -1.5 & -0.5 & 0.5 & 1.5 & 2.5 & 3.5 \\
17 & -8 & -7 & -6 & -5 & -4 & -3 & -2 & -1 & 0 & 1 & 2 & 3 \\
18 & -8.5 & -7.5 & -6.5 & -5.5 & -4.5 & -3.5 & -2.5 & -1.5 & -0.5 & 0.5 & 1.5 & 2.5 \\
19 & -9 & -8 & -7 & -6 & -5 & -4 & -3 & -2 & -1 & 0 & 1 & 2 \\
20 & -9.5 & -8.5 & -7.5 & -6.5 & -5.5 & -4.5 & -3.5 & -2.5 & -1.5 & -0.5 & 0.5 & 1.5 \\
\hline
\end{tabular}

Remarques: $\quad q_{1}(n, n-1-\xi)=-q_{1}(n, \xi)$.

La table a été calculée à l'aide de la formule:

$$
q_{1}=\xi-\frac{1}{2}(n-1) \text {. }
$$


II. Taple des valeurs de $q_{2}(n, \xi)$.

\begin{tabular}{|c|c|c|c|c|c|c|c|c|c|c|c|c|}
\hline$m, \xi$ & 0 & 1 & 2 & 3 & 4 & 5 & 6 & 7 & 8 & 9 & 10 & 11 \\
\hline 3 & 0.5 & -1 & 0.5 & & & & & & & & & \\
\hline 4 & $1 \cdot 5$ & $-1 \cdot 5$ & $-1 \cdot 5$ & 1.5 & & & & & & & & \\
\hline 5 & 3 & $-1 \cdot 5$ & -3 & -1.5 & 3 & & & & & & & \\
\hline 0 & 5 & -1 & -4 & -4 & -1 & 5 & & & & & & \\
\hline 7 & $7 \cdot 5$ & 0 & $-4 \cdot 5$ & -6 & -4.5 & 0 & $7 \cdot 5$ & & & & & \\
\hline 8 & 10.5 & 1.5 & -4.5 & $-7 \cdot 5$ & -7.5 & -4.5 & $1 \cdot 5$ & 10.5 & & & & \\
\hline 9 & 14 & 3.5 & -4 & -8.5 & -10 & -8.5 & -4 & 3.5 & 14 & & & \\
\hline 10 & 18 & 6 & -3 & -9 & -12 & -12 & -9 & -3 & 6 & 18 & & \\
\hline 11 & $22 \cdot 5$ & 9 & -1.5 & -9 & $-13 \cdot 5$ & -15 & $-13 \cdot 5$ & -9 & -1.5 & 9 & $22 \cdot 5$ & \\
\hline 12 & $27 \cdot 5$ & $12 \cdot 5$ & 0.5 & $-8 \cdot 5$ & $-14 \cdot 5$ & $-17 \cdot 5$ & $-17 \cdot 5$ & $-14 \cdot 5$ & $-8 \cdot 5$ & 0.5 & $12 \cdot 5$ & $27 \cdot 5$ \\
\hline 13 & 33 & $16 \cdot 5$ & 3 & $-7 \cdot 5$ & -15 & $-19 \cdot 5$ & -21 & $-19 \cdot 5$ & -15 & $-7 \cdot 5$ & 3 & 16.5 \\
\hline 14 & 39 & 21 & 6 & -6 & -15 & -21 & -24 & -24 & -21 & -15 & -6 & 6 \\
\hline 15 & $45 \cdot 5$ & 26 & 9.5 & -4 & $-14 \cdot 5$ & -22 & -26.5 & -28 & -26.5 & -22 & $-14 \cdot 5$ & -4 \\
\hline 16 & $52 \cdot 5$ & $31 \cdot 5$ & 13.5 & -1.5 & -13.5 & $-22 \cdot 5$ & $-28 \cdot 5$ & $-31 \cdot 5$ & $-31 \cdot 5$ & -28.5 & $-22 \cdot 5$ & $-13 \cdot 5$ \\
\hline 17 & 60 & $37 \cdot 5$ & 18 & 1.5 & -12 & $-22 \cdot 5$ & -30 & $-34 \cdot 5$ & -36 & $-34 \cdot 5$ & -30 & $-22 \cdot 5$ \\
\hline 18 & 68 & 44 & 23 & 5 & -10 & -22 & -31 & -37 & -40 & -40 & -37 & -31 \\
\hline 19 & 76.5 & 51 & $28 \cdot 5$ & 9 & $-7 \cdot 5$ & -21 & $-31 \cdot 5$ & -39 & $-43 \cdot 5$ & -45 & -43.5 & -39 \\
\hline 20 & $85 \cdot 5$ & $58 \cdot 5$ & $34 \cdot 5$ & $13 \cdot 5$ & -4.5 & $-19 \cdot 5$ & -31.5 & -40.5 & -46.5 & $-49 \cdot 5$ & -49.5 & -46.5 \\
\hline
\end{tabular}

Remarque :

$g_{2}(n, n-1-\xi)=q_{2}(n, \xi)$.

La table a été calculée à l'aide de la formule :

$q_{2}=\frac{1}{4}(2-n)_{2}+\frac{3}{2}(2-n) \xi+\frac{3}{2}(\xi) 2$.

III. Table Des valeurs De $q_{3}(n, \xi)$.

\begin{tabular}{|r|l|l|l|l|l|l|l|l|l|l|l|}
\hline$n, \xi$ & 0 & 1 & 2 & 3 & 4 & 5 & 6 & 7 & 8 & 9 & 10 \\
\hline 4 & $-0 \cdot 75$ & $2 \cdot 25$ & $-2 \cdot 25$ & $0 \cdot 75$ & & & & & & \\
5 & -3 & 6 & -0 & -6 & 3 & & & & & \\
6 & $-7 \cdot 5$ & $10 \cdot 5$ & 6 & -6 & $-10 \cdot 5$ & $7 \cdot 5$ & & & & \\
7 & -15 & 15 & 15 & 0 & -15 & -15 & 15 & & & \\
8 & $-26 \cdot 25$ & $18 \cdot 75$ & $26 \cdot 25$ & $11 \cdot 25$ & $-11 \cdot 25$ & $-26 \cdot 25$ & $-18 \cdot 75$ & $26 \cdot 25$ & & \\
9 & -42 & 21 & 39 & 27 & 0 & -27 & -39 & -21 & 42 & \\
10 & -63 & 21 & $52 \cdot 5$ & $46 \cdot 5$ & 18 & -18 & $-46 \cdot 5$ & $-52 \cdot 5$ & -21 & 63 & \\
11 & -90 & 18 & 66 & 69 & 42 & 0 & -42 & -69 & -66 & -18 & 90 \\
12 & $-123 \cdot 75$ & $11 \cdot 25$ & $78 \cdot 75$ & $93 \cdot 75$ & $71 \cdot 75$ & $26 \cdot 25$ & $-28 \cdot 25$ & $-71 \cdot 25$ & $-93 \cdot 75$ & $-78 \cdot 75$ & $-11 \cdot 25$ \\
13 & -165 & 0 & 90 & 120 & 105 & 60 & 0 & -60 & -105 & -120 & -90 \\
14 & $-214 \cdot 5$ & $-16 \cdot 5$ & 99 & 147 & $142 \cdot 5$ & $100 \cdot 5$ & 36 & -36 & $-100 \cdot 5$ & $-142 \cdot 5$ & -147 \\
15 & -273 & -39 & 105 & 174 & 183 & 147 & 81 & 0 & -81 & -147 & -183 \\
16 & $-341 \cdot 25$ & $-68 \cdot 25$ & $107 \cdot 25$ & $200 \cdot 25$ & $225 \cdot 75$ & $198 \cdot 75$ & $134 \cdot 25$ & $47 \cdot 25$ & $-47 \cdot 25$ & $-134 \cdot 25$ & $-198 \cdot 75$ \\
17 & -420 & -105 & 105 & 225 & 270 & 255 & 195 & 105 & 0 & -105 & -195 \\
18 & -510 & -150 & $97 \cdot 5$ & $247 \cdot 5$ & 315 & 315 & $262 \cdot 5$ & $172 \cdot 5$ & 60 & -60 & $-172 \cdot 5$ \\
19 & -612 & -204 & 84 & 267 & 360 & 378 & 336 & 249 & 132 & 0 & -132 \\
20 & $-726 \cdot 75$ & $-267 \cdot 75$ & $63 \cdot 75$ & $282 \cdot 75$ & $404 \cdot 25$ & $443 \cdot 25$ & $414 \cdot 75$ & $333 \cdot 75$ & $215 \cdot 25$ & $74 \cdot 25$ & $-74 \cdot 25$ \\
& & & & & & & & & & \\
\hline
\end{tabular}

Remarque :

La table a été ealculée par la formule :

$$
q_{3}=\frac{1}{8}(3-n)_{3}+\frac{3}{2}(3-n)_{3} \xi+\frac{15}{4}(3-n)(\xi)_{2}+\frac{5}{2}(\xi)_{3} \cdot
$$


IV. Table Des valeurs DE $q_{4}(n, \xi)$.

\begin{tabular}{|c|c|c|c|c|c|c|c|c|c|c|c|}
\hline$n, \xi$ & 0 & 1 & 2 & 3 & 4 & 5 & 6 & 7 & 8 & 9 & 10 \\
\hline 5 & 1.5 & -6 & 9 & - & 1.5 & & & & & & \\
\hline 6 & $7 \cdot 5$ & $-22 \cdot 5$ & 15 & 15 & -22.5 & $7 \cdot 5$ & & & & & \\
\hline 7 & 22.5 & -52.5 & $7 \cdot 5$ & 45 & $7 \cdot 5$ & -52.5 & $22 \cdot 5$ & & & & \\
\hline 8 & 52.5 & -97.5 & $-\quad 22.5$ & $67 \cdot 5$ & $67 \cdot 5$ & -22.5 & -97.5 & $52 \cdot 5$ & & & \\
\hline 9 & 105 & -157.5 & $-\quad 82.5$ & $67 \cdot 5$ & 185 & 675 & -82.5 & $-157 \cdot 5$ & 105 & & \\
\hline 10 & 189 & -231 & $-178 \cdot 5$ & $31 \cdot 5$ & 189 & 189 & 31.5 & -178.5 & -231 & 189 & \\
\hline 11 & 315 & -315 & -315 & $-\quad 52.5$ & 210 & 315 & 210 & $-\quad 52.5$ & -315 & -315 & 315 \\
\hline 12 & 495 & -405 & -495 & -195 & 180 & 420 & 420 & 180 & -195 & -495 & -405 \\
\hline 13 & 742.5 & -495 & -720 & -405 & $82 \cdot 5$ & 480 & 630 & 480 & $82 \cdot 5$ & -405 & -720 \\
\hline 14 & $1072 \cdot 5$ & -577.5 & -990 & -690 & -97.5 & $472 \cdot 5$ & 810 & 810 & $472 \cdot 5$ & $-\quad 97 \cdot 5$ & -690 \\
\hline 15 & 1501.5 & $-643 \cdot 5$ & $-1303 \cdot 5$ & -1056 & $-373 \cdot 5$ & $376 \cdot 5$ & $931 \cdot 5$ & 1134 & 931.5 & 376.5 & -373.5 \\
\hline 16 & $2047 \cdot 5$ & $-682 \cdot 5$ & $-1657 \cdot 5$ & -1507 '5 & $-757 \cdot 5$ & $172 \cdot 5$ & $967 \cdot 5$ & $1417 \cdot 5$ & $1417 \cdot 5$ & $967 \cdot 5$ & $172 \cdot 5$ \\
\hline 17 & 2730 & $-682^{\prime} 5$ & $-2047 \cdot 5$ & $-2047 \cdot 5$ & -1260 & -157.5 & 892.5 & $1627 \cdot 5$ & 1890 & $1627 \cdot 5$ & 892.5 \\
\hline 18 & 3570 & -630 & -2467.5 & $-2677 \cdot 5$ & -1890 & -630 & $682 \cdot 5$ & 1732.5 & 2810 & 2310 & 1732.5 \\
\hline 19 & 4590 & -510 & -2910 & -3397.5 & -2655 & -1260 & 315 & $1702 \cdot 5$ & 2640 & 2970 & 2640 \\
\hline 20 & 5814 & -306 & -3366 & -4206 & -3561 & -2061 & -231 & 1509 & 2844 & 3564 & 3564 \\
\hline
\end{tabular}

Remarque :

$$
q(n, \xi)=q(n, n-1-\xi)
$$

La table a été calculée à l'aide de la formule :

$$
q_{4}(\xi)=\frac{1}{16}\left[(4-n)_{4}+2 j(4-n)_{3} \xi+90(4-n):(\xi) \geq+140(4-n)(\xi)_{3}+70(\xi)_{4}\right] .
$$

\begin{tabular}{|c|c|c|c|c|c|c|c|c|c|c|c|}
\hline$n, \xi$ & 0 & 1 & 2 & 3 & 4 & 5 & 6 & 7 & 8 & 9 & 10 \\
\hline 6 & $-\quad 3.75$ & $18 \cdot 75$ & $-37 \cdot 5$ & $37 \cdot 5$ & $-\quad 18.75$ & $3 \cdot 75$ & & & & & \\
\hline 7 & $22 \cdot 5$ & 90 & $-112 \cdot 5$ & 0 & $112 \cdot 5$ & 90 & 22.5 & & & & \\
\hline 8 & $-\quad 78.5$ & $258 \cdot 75$ & $-191 \cdot 25$ & $-168 \cdot 75$ & $188 \cdot 75$ & $191 \cdot 25$ & $258 \cdot 75$ & $78 \cdot 75$ & & & \\
\hline 9 & -210 & $577 \cdot 5$ & -210 & $-472 \cdot 5$ & 0 & 472.5 & 210 & $577 \cdot 5$ & 210 & & \\
\hline 10 & $-472 \cdot 5$ & $1102 \cdot 5$ & -78.75 & $-866 \cdot 25$ & -472.5 & $472 \cdot 5$ & $866 \cdot 25$ & $78 \cdot 75$ & $-1102 \cdot 5$ & $472 \cdot 5$ & \\
\hline 11 & -945 & 1890 & 315 & -1260 & -1260 & 0 & 1260 & 1260 & -315 & -1890 & 945 \\
\hline 12 & $-1732 \cdot 5$ & $299: 5$ & $1102 \cdot 5$ & $-1522 \cdot 5$ & -2310 & -1050 & 1050 & 2310 & $1522 \cdot 5$ & $-1102 \cdot 5$ & $-2992 \cdot 5$ \\
\hline 13 & -2970 & 4455 & 2430 & -1485 & -3510 & -2700 & 0 & 2700 & 3510 & 1485 & -2430 \\
\hline I4 & $-4826 \cdot 25$ & $6311 \cdot 25$ & 4455 & -945 & $-4691 \cdot 25$ & -4893.25 & -2025 & 2025 & $4893 \cdot 25$ & $4691 \cdot 25$ & 945 \\
\hline 15 & $-7507 \cdot 5$ & 8580 & $7342 \cdot 5$ & 330 & $-5632 \cdot 5$ & -7500 & $-5062 \cdot 5$ & 0 & $5062 \cdot 5$ & 7500 & $5632 \cdot 5$ \\
\hline 16 & $-11261 \cdot 25$ & $11261 \cdot 25$ & $11261 \cdot 25$ & $2598 \cdot 75$ & $-6063 \cdot 75$ & $-10316 \cdot 25$ & $-9056 \cdot 25$ & $-8543 \cdot 75$ & $3543 \cdot 75$ & $9056 \cdot 25$ & $10316 \cdot 25$ \\
\hline 17 & -16380 & $14332 \cdot 5$ & 16380 & $6142 \cdot 5$ & -5670 & $-13072 \cdot 5$ & -13860 & $-866 z \cdot 5$ & 0 & $8662 \cdot 5$ & 13860 \\
\hline 18 & -23205 & 17745 & $22863 \cdot 75$ & $11261 \cdot 25$ & -4095 & -15435 & $-19241 \cdot 25$ & $-15303 \cdot 75$ & -5775 & 5775 & $15303 \cdot 75$ \\
\hline 19 & -32130 & 21420 & 30870 & 18270 & -945 & -17010 & -24885 & -23310 & -13860 & 0 & 13860 \\
\hline 20 & -43605 & 25245 & 40545 & 27495 & $4207 \cdot 5$ & $-17347 \cdot 5$ & $-30397 \cdot 5$ & $-32422 \cdot 5$ & -24210 & -8910 & 8910 \\
\hline
\end{tabular}

V. TABle des valeurs de $q_{j}(n, \xi)$.

Remarques: On a $q_{5}(n, \xi)=-q_{5}(n, n-1-\xi)$.

Les valeurs ci-dessus ont été calculées a l'aide de la formule $q_{5}(n, \xi)=\frac{1}{3 \frac{1}{2}}(5-n)_{5}+\frac{15}{16}(5-n)_{4} \xi+\frac{105}{16}(5-n)_{3}(\xi)_{2}+\frac{35}{2}(5-n)_{2}(\xi)_{3}+3_{16}^{315}(5-n)(\xi)_{4}+\frac{83}{8}(\xi)_{5}$ 
VI. TABLE DES VALEURS DE $\sum_{k=0}^{n}\left[q_{m}(n, \xi)\right] *$

\begin{tabular}{|r|l|l|l|c|c|}
\hline$n, m$ & 1 & 2 & 3 & 4 & 5 \\
\hline 2 & $0 \cdot 5$ & & & & \\
3 & 2 & $1 \cdot 5$ & & & \\
4 & 5 & 9 & $11 \cdot 25$ & & \\
5 & 10 & $31 \cdot 5$ & 90 & $157 \cdot 5$ & \\
6 & $17 \cdot 5$ & 84 & 405 & 1575 & $3543 \cdot 75$ \\
7 & 28 & 189 & 1350 & $8662 \cdot 5$ & 42525 \\
8 & 42 & 378 & $3712 \cdot 5$ & 34650 & $276412 \cdot 5$ \\
9 & 60 & 693 & 8910 & $112612 \cdot 5$ & 1289925 \\
10 & $82 \cdot 5$ & 1188 & 19305 & 315315 & $4837218 \cdot 75$ \\
11 & 110 & $1930 \cdot 5$ & 38610 & $788287 \cdot 5$ & 15479100 \\
12 & 143 & 3003 & $72393 \cdot 75$ & 1801800 & 43857450 \\
13 & 182 & $4504 \cdot 5$ & 128700 & 3828825 & 112736300 \\
14 & $227 \cdot 5$ & 6552 & 218790 & 7657650 & $267843637 \cdot 5$ \\
15 & 280 & 9282 & 359020 & 14549535 & 595208250 \\
16 & 340 & 12852 & 566865 & 26453700 & 1249937325 \\
17 & 408 & 17442 & 872100 & 46293975 & 2499874650 \\
18 & $484 \cdot 5$ & 23256 & 1308150 & 78343650 & $4791426412 \cdot 5$ \\
19 & 570 & $30523 \cdot 5$ & 1918620 & 128707425 & 8845710300 \\
20 & 665 & 39501 & $2758016 \cdot 25$ & 205931880 & 15795911250 \\
\hline
\end{tabular}

Remarque : La table a été calculée à l'aide de la formule suivante :

$$
\Sigma\left(q_{m}\right)^{2}=\frac{n}{(2 m+1) 2^{2 m}} \prod_{s=1}^{m+1}\left(n^{2}-s^{2}\right) .
$$

